\title{
Why the Ultimate Argument for Scientific Realism Ultimately Fails ${ }^{1}$
}

\author{
Moti Mizrahi $^{2}$
}

\begin{abstract}
In this paper, I argue that the ultimate argument for Scientific Realism, also known as the No-Miracles Argument (NMA), ultimately fails as an abductive defence of Epistemic Scientific Realism (ESR), where (ESR) is the thesis that successful theories of mature sciences are approximately true. The NMA is supposed to be an Inference to the Best Explanation (IBE) that purports to explain the success of science. However, the explanation offered as the best explanation for success, namely (ESR), fails to yield independently testable predictions that alternative explanations for success do not yield. If this is correct, then there seems to be no good reason to prefer (ESR) over alternative explanations for success.
\end{abstract}

Keywords: inference to the best explanation; no-miracles argument; predictive success; scientific realism; ultimate argument for scientific realism

\section{Introduction}

There are many versions of Scientific Realism in the literature. Usually, Scientific Realism is taken to include one or more of the following theses (Psillos, 2006, p. 135):

(MSR) The Metaphysical Thesis: The world has a definite and mind-independent structure.

(SSR) The Semantic Thesis: Scientific theories are truth-conditioned descriptions of their intended domain. Hence, they are capable of being true or false. The theoretical terms featuring in theories have putative factual reference. So if scientific theories are true, the unobservable entities they posit populate the world.

(ESR) The Epistemic Thesis: Mature and predictively successful scientific theories are well-confirmed and approximately true. So entities posited by them, or, at any rate entities very similar to those posited, inhabit the world (Cf. Psillos, 1999).

The most prominent argument in support of (ESR) is known as the No-Miracles Argument (NMA). Van Fraassen (1980, p. 39) has dubbed this argument "the ultimate argument for realism," and Musgrave (1988) has written a paper, entitled "The Ultimate Argument for Scientific Realism,” defending the NMA. But the argument goes back to Putnam (1975, p. 73), who put it as follows: Scientific Realism "is the only philosophy that doesn't make the success of science a miracle.”

The NMA is usually construed as an abductive defence of (ESR). Abductive reasoning, also known as Inference to the Best Explanation (IBE), is a form of non-deductive reasoning. Harman (1965, p. 89) defines IBE as inference "from the premise that a given hypothesis would provide a 'better' explanation for the evidence than would any other hypothesis, to the conclusion that the given hypothesis is true.” The form of IBE, then, is roughly the following:

\footnotetext{
${ }^{1}$ Forthcoming in Studies in History and Philosophy of Science.

${ }^{2}$ Philosophy Department, St. John’s University, 8000 Utopia Parkway, Queens, NY 11439. Email: motimizra@gmail.com.
} 
Phenomenon $P$.

The best explanation for $P$ is hypothesis $H$.

Therefore, (probably) $H$.

Similarly, Psillos (2002, p. 614) formulates IBE as follows:

$D$ is a collection of data (facts, observations, givens).

$H$ explains $D$ (would, if true, explain $D$ ).

No other hypothesis can explain $D$ as well as $H$ does.

Therefore, $H$ is probably true.

And Iranzo (2007, p. 341) puts IBE schematically as follows:

$e$ is a collection of data, facts, observations, ...

$h$ explains $e$ (if $h$ were true, $e$ would be a matter of course)

no other hypothesis explains $e$ as well as $h$ does

Therefore, $h$ is true. ${ }^{3}$

Accordingly, the NMA may be reconstructed as follows:

(NMA1) The enterprise of science is enormously successful.

(NMA2) The best explanation for this success is (ESR).

(NMA3) Therefore, (ESR) is probably true (Cf. Kukla, 1998, p. 12).

The intuitively plausible idea underlying the NMA is the following: if scientific theories are false, how is it that they are so successful? "Success" here means explanatory success, i.e., that mature scientific theories explain a broad range of natural phenomena. More importantly, "success" also means predictive success, i.e., that mature scientific theories make predictions that are borne out by experimental and observational tests. As Kukla (1998, p. 12) puts it, "our scientific theories enable us to make significantly more correct predictions than we could make without them."

When dealing with arguments of the form IBE, there are at least three obvious questions that one might ask: ${ }^{4}$

\footnotetext{
${ }^{3}$ Iranzo (2007) distinguishes between IBE as a heuristic procedure and IBE as an epistemic rule. "According to the former, IBE equates to a search strategy for abductive solutions, that is, for potential explanations" (Iranzo, 2007, p. 340). According to the latter, IBE is truth-conducive: "true or highly probable explanations are favoured while false, or low-probability ones, are discarded" (Iranzo, 2007, p. 341). The epistemological challenge, according to Iranzo (2007, p. 344), is the following: "why should we think that those explanations that afford explanatory coherence are true, or highly likely?” For Niiniluoto (2004), the best explanation is the hypothesis that has the highest degree of confirmation. For Psillos (2002), the best explanation is the hypothesis that fits better with background knowledge. In what follows, I will not be concerned with the epistemological challenge to IBE, but with the NMA in particular. ${ }^{4}$ There are other questions one could ask about IBE. For example, is it possible that there is an explanation that has been overlooked? See Stanford (2006). For present purposes, however, I will focus on questions (a)-(c).
} 
(a) Are there alternative explanations for the phenomena in question?

(b) If so, which of these explanations counts as "the best"?

(c) What are the criteria for selecting "the best" explanation?

As far as (a) is concerned, anti-realists have offered alternative explanations for the success of science. ${ }^{5}$ For example, van Fraassen (1980, p. 40) provides the following explanation, which is based on an evolutionary analogy:

I claim that the success of current scientific theories is no miracle. It is not even surprising to the scientific (Darwinist) mind. For any scientific theory is born into a life of fierce competition, a jungle red in tooth and claw. Only the successful theories survive - the ones which in fact latched on to actual regularities in nature.

Van Fraassen's point seems to be that a theory can be empirically adequate, and hence latch on to the observable regularities in nature, without being true. For a constructive empiricists like van Fraassen, the scientific competition between theories hinges on which theory accurately describes the observable world, not on which theory is actually true. Thus, it would not be miraculous for science to arrive at an empirically adequate, scientifically successful, yet false theory.

Leplin (1997) distinguishes two types of anti-realist explanations for the success of science: (i) those that appeal to evolutionary mechanisms for theory selection, like van Fraassen's evolutionary explanation, and (ii) those that regard a theory's empirical adequacy already to be an explanation of its success. He argues that both anti-realist strategies fail to achieve their purpose, so the only explanation for scientific success is (approximate) truth. The problem with (i), according to Leplin, is that the question answered by evolutionary epistemology is not the same as the question answered by (approximate) truth. Evolutionary epistemology explains how we come to be in possession of successful theories, but it does not explain why it is that these theories, which happen to be the ones we possess, are successful. On the other hand, (ESR) cites (approximate) truth as a property of successful theories that accounts for why they are successful. (ESR) does not explain how we come to possess these successful theories. In other words, if we ask "Why is quantum mechanics successful?", then "Because it is true" seems to be a potential explanation, whereas "Because its adoption is the result of a process of Darwinian selection for success" does not.

In reply, Kukla (1998, p. 22) distinguishes between the following alternative explanations for the success of science:

(SS) Strong Surrealism: Mature scientific theories are empirically equivalent to true theories.

(WS) Weak Surrealism: The observable world behaves as if our mature scientific theories are true.

According to (WS), the truth of our observable predictions is explained by the facts that these predictions are consequences of mature scientific theories, and the world behaves as if mature

\footnotetext{
${ }^{5}$ For anti-realist explanations for the success of science, see also Fine (1986), Stanford (2000), and Lyons (2003).
} 
scientific theories were true. According to Kukla (1998, p. 23), since this claim goes beyond what is contained in the explanandum (i.e., the success of science), (WS) is a viable alternative explanation for the success of science. ${ }^{6}$

Since anti-realists do offer alternative explanations for the success of science, questions (b) and (c) must be addressed. This is the aim of this paper. To simplify matters, I will only consider (ESR) and one alternative explanation, namely (WS). For present purposes, then, the crucial question is how to select between (ESR) and (WS). For the NMA to work, it must be the case that (ESR) is better than (WS), as an explanation for the success of science, or that (WS) cannot explain success as well as (ESR) does. But is that the case? My overall argument is the following:

(1) In IBE, if two equally plausible explanations yield the same independently testable predictions, then there is no good reason to prefer one over the other.

(2) (ESR) and (WS) are two equally plausible explanations for the success of science that yield the same independently testable predictions.

(3) Therefore, there is no good reason to prefer (ESR) over (WS).

To be clear, this argument does not establish that (ESR) is false, unlikely, or implausible. Rather, it simply shows that (ESR) cannot be defended on abductive grounds. That is to say, it shows that there is no good reason to prefer (ESR) over (WS) as the best explanation for the success of science. The following sections flesh out each premise of this argument.

\section{IBE and predictive power}

IBE is about selecting the best explanation among potential explanations. How do we choose among potential explanations? According to Lipton (2004), IBE is a two-step process, which filters out potential explanations:

- The first step filters out the implausible explanations. At this stage, one considers all the plausible potential explanations and rules out the implausible ones.

- The second step filters out the lower ranking plausible explanations. At this stage, one examines the plausible potential explanations that have passed through the first filter, and ranks them in order to select the top ranking explanation as the best explanation. ${ }^{7}$

For Lipton, explanatory goodness, or what he calls “loveliness," must be distinguished from likeliness. Loveliness is a matter of potential understanding, i.e., a lovely explanation is one that, if it were true, would provide understanding of the relevant phenomena. To this, Bird (2010, p. 346) adds the following qualifications concerning the second step:

\footnotetext{
${ }^{6}$ Cf. Stanford (2000). Stanford (2000, p. 275) offers an alternative explanation for the success of science according to which the success of any theory $T$ is explained by its predictive similarity to the "true theoretical account of the relevant domain.” Cf. Psillos (2001).

${ }^{7}$ Cf. Iranzo (2007, p. 340), who puts this "double-filter" model of IBE in terms of a distinction between generation and selection. According to Iranzo (2007, p. 340), first "there is an explicit search for alternative potential explanations which fit with the evidence," and then there is a "comparison among them [...] concerning the degree [to which] they satisfy some explanatory desiderata.” See also Bird (2007).
} 
(Q1) For the best explanation to be inferred it must be significantly better than its nearest rival. If two competing explanations are both good enough, and one is slightly better than the other, our faith in that slightly better one must be slim.

(Q2) For the best explanation to be inferred it should normally, considered on its own, be a sufficiently good explanation of enough evidence. If our best explanation is a weak explanation even of a large quantity of data, or explains only a limited amount of evidence well, then that is some reason to doubt that it is the actual explanation.

As Bird (2010, p. 346) points out, important questions may be raised about each step of IBE. Concerning the first step, since it filters out logically possible explanations, can we be confident that the actual explanation is not filtered out as well? This is what Lipton (2004, p. 152) calls the problem of "Underconsideration." If the actual explanation was filtered out in the first step, simply because scientists failed to think of it, then the ranking in the second step will be of no use.

Even if we assume that the actual explanation has made it to the second step, there are two further problems. The first is what Lipton calls "Hungerford's objection," according to which the loveliness of explanations may be too subjective to have any relationship to the truth. The second is what Lipton calls "Voltaire's objection," according to which advocates of IBE have an unjustified Panglossian faith that the actual world is the loveliest of all possible worlds. Even if loveliness is objective, there will be possible worlds in which it does not correlate with truth. If that is correct, then why think that truth and loveliness correlate in our world?

Because of these and other problems, anti-realists usually deny that IBE is a legitimate form of inference in science. For instance, according to van Fraassen (1980, p. 143), IBE "is a rule that selects the best among the historically given hypotheses. We can watch no contest of the theories we have so painfully struggled to formulate, with those no one has proposed. So our selection may well be the best of a bad lot." 8

Even if the above questions can be answered, and the aforementioned objections can be met, ${ }^{9}$ proponents of IBE still have to say on what grounds the ranking in the second step is based. In other words, what are the criteria for ranking plausible explanations from best to worst? Proponents of IBE have proposed several criteria of selection. For example:

Unification: As a general rule of thumb, choose the explanation that explains the most and leaves the least unexplained things. ${ }^{10}$

Testability: As a general rule of thumb, choose the explanation that yields independently testable predictions. ${ }^{11}$

\footnotetext{
${ }^{8}$ See also Muller (2008, p. 143): an inference "going from observable behaviour to unobservable mental states, smacks too much of an Inference-to-the-Best-Explanation (IBE), which is a mode of inference that Van Fraassen is very critical about, in particular when it concerns an explanandum about observables only and an explanans which is also about unobservable.”

${ }^{9}$ Another objection against the NMA, which I will not discuss in this paper, is that it is viciously circular. See Lipton (2000, pp. 194-195) and Psillos (1999, chap. 4). See also Iranzo (2008) and Newman (2010).

10 See, e.g., Kitcher (1981). Cf. Newman (2010).

${ }^{11}$ See, e.g., Zahar (1973), Kitcher (1983), and Leplin (1997). Cf. Newman (2010).
} 
Coherence: As a general rule of thumb, choose the explanation that is consistent with background knowledge. ${ }^{12}$

Simplicity: As a general rule of thumb, choose the least complicated explanation, i.e., the one that posits the least causal sequences and entities, and that goes beyond the evidence the least. ${ }^{13}$

But how do we select the best explanation from a set that contains plausible explanations that are equally good in terms of the criteria listed above? Suppose we have two competing explanations that have passed the first step that filters out implausible explanations. What would separate these two explanations? In the next section, I argue that only independent testing of predictions can separate the two explanations. Then I will argue that (ESR) and (WS) are two plausible explanations for success, which yield the same predictions. If this is so, then there is no reason to prefer (ESR) over (WS), as an explanation for the success of science, when these two explanations are judged by abductive lights.

\section{Selecting the best of two plausible explanations}

To see why the only way to choose between equally plausible explanations is to independently test the predictions they yield, consider the following scenario:

A man is found in a cabin in a remote forest, with all the doors and windows securely locked from the inside, hanging dead from a noose. A suicide note lies on the table nearby. What would best explain this state of affairs? ${ }^{14}$

Although the man's death seems to be an apparent case of suicide, there are other plausible explanations. Consider the following alternative explanation:

(AE) The man was rehearsing a drama about suicide, had locked the doors for privacy, and things had gone terribly wrong.

(AE) is consistent with the evidence. The evidence does not leave suicide as the only plausible explanation. Of these two plausible explanations, then, which one counts as "the best"?

Since both (AE) and suicide seem to be plausible explanations for the man's death, it seems that the only way to choose between these two explanations is to test them. (AE) yields several predictions that can be tested independently. For example, the man should own a script, perhaps have been a member of a theatre group, or told his friends that he was involved in a play. Whether or not the man was a member of a theatre group, for instance, can be checked independently of (AE), i.e., by calling local theatre groups and checking their members lists.

Like (AE), suicide also explains the facts before us and allows us to make testable predictions. For example, a potential prediction is that the man was depressed and under stress. Whether or not the man was under stress, for instance, can be checked independently of the

\footnotetext{
${ }^{12}$ See, e.g., Kosso (1992). Cf. Newman (2010).

${ }^{13}$ See, e.g., Sober (1988), (1994), and (1998). Cf. Newman (2010).

${ }^{14}$ This example is adapted from Baggini and Fosl (2010, p. 43).
} 
suicide explanation, i.e., by checking his medical record, doctors' visits, any prescriptions he may have been taking, talking to his spouse and friends, etc.

Selecting the best explanation for the man's death, then, will depend on independently testing the predictions that each explanation yields. Suppose we find out that the man was recently fired from his job, filed for bankruptcy, and got divorced. These findings would lend support to the suicide explanation. On the other hand, suppose we find out that the man was an actor, and that he had told his friends that he doesn't want to be bothered this weekend because he needs to rehearse for an important role in a major play. These findings would lend support to (AE) as an explanation for the man's death. In any case, it would be the independent testing of predictions that would help us choose between these two plausible explanations.

Now consider an example from science. During the 1920s, Edwin Hubble used the newly constructed Mount Wilson telescope in California to resolve individual variable stars within the Andromeda galaxy. This allowed him to demonstrate that Andromeda lay well outside the Milky Way. This opened the way to the view that the Milky Way is just one of a vast (perhaps infinite) number of galaxies throughout the universe. Meanwhile, Alexander Friedmann discovered in 1922 the class of cosmological models, now known as Friedmann cosmologies, which are models of an expanding universe. They were also later discovered independently by Georges Lemaître, who recognized that they predicted an initial time when the universe was of zero size, which he called the "primeval atom."

Subsequently, in 1929, Hubble and Milton Humason measured the redshifts of many nearby galaxies, and they were able to show that the vast majority of them were receding from the Milky Way. They also found that the rate of recession increased in proportion to the distance of the galaxy. This expansion law, known as Hubble expansion, has been confirmed and extended to distant galaxies. According to this law, all observers in the universe see that galaxies recede radially with velocity proportional to their distance. The Hubble law is thus compatible with the cosmological principle, which states that there are no special observers in the universe.

At that point, there were two plausible explanations for the expansion of the universe. The first was the big-bang model, according to which the universe underwent an explosion from a point of initial singularity. The second was the steady-state model, according to which the universe has always been expanding (no explosion event) and its constant mean density is maintained by the continuous creation of matter.

Then, in the mid-1940s, George Gamow realized that the young big bang would have been such a hot environment that nuclear reactions would have been important, and sought a cosmological theory of the nucleosynthesis process leading to the observed abundances of different chemical elements in the universe. He found that the lighter elements, particularly hydrogen and helium-4, would indeed be a consequence of the big bang model. The majority of heavier elements would later be shown, by Fred Hoyle and others, to be created within stars. Based on this work, Ralph Alpher and Robert Herman predicted in 1948 the existence of relic radiation left over from the hot early phase, which would suffuse the universe. However, Alpher and Herman didn't think their predicted radiation was detectable, and their ideas were largely forgotten for over a decade. 
This radiation, now known as the Cosmic Microwave Background radiation (CMB), was fortuitously discovered in 1965 by Arno Penzias and Robert Wilson. In 1960, Bell Labs built a giant antenna in Holmdel, New Jersey. It was part of a satellite transmission system called Echo. By collecting and amplifying weak radio signals bounced off large metallic balloons in the atmosphere, it could send signals across long distances. When Penzias and Wilson began to use the antenna as a telescope they found there was a background "noise" (much like static in a radio). This annoyance was a uniform signal in the microwave range, seeming to come from all directions. They checked everything to rule out the source of the excess radiation. After ruling out all possible sources, they concluded it was neither the machine nor random noise causing the radiation. At first, Penzias and Wilson didn't know what to make of their discovery. Around the same time, Robert Dicke had been working on the big bang. Dicke speculated that, if there had been an explosion, the residue should by now take the form of a low-level background radiation throughout the universe. Shortly after, Penzias and Wilson got in touch with his lab.

As Leplin points out (1997, p. 94), the CMB is an independently testable prediction of the big-bang model. Furthermore, the discovery of the CMB makes sense from the vantage point of the big-bang model, but not from the vantage point of the steady-state model, and that is why it counts in favour of the former. Now, for present purposes, the question is the following: Can we choose between (ESR) and (WS) in the same way that cosmologists preferred the big-bang model over the steady-state model? Do these two alternative explanations for the success of science yield independently testable predictions, which, if borne out, would give us good reasons to prefer one over the other? In the next section, I argue that there are no independently testable predictions made by (ESR) that would clearly favour it over (WS).

\section{4. (ESR) and independently testable predictions}

Does (ESR) yield independently testable predictions? Recall that we are looking for predictions of the following sort:

(P1) If suicide is the best explanation for the man's death, then we would expect to find a motive for suicide, e.g., depression as a result of divorce, bankruptcy, etc.

(P2) If (AE) is the best explanation for the man's death, then we would expect to find a script he was rehearsing.

(P3) If the steady-state model is the best explanation for the expansion of the universe, then we would expect to find that the density of matter in the universe remains constant as the universe expands.

(P4) If the big-bang model is the best explanation for the expansion of the universe, then we would expect to find relic radiation left over from earlier stages of the hot big bang.

Similarly, if (ESR) is the best explanation for the success of science, then there should be certain things we would expect to find that would make sense only from the vantage point of (ESR). In that respect, note that the following "prediction" will not do: 
(P5) If (ESR) is the best explanation for the success of science, then we would expect science to be successful.

(P5) cannot help us choose between (ESR) and (WS). First, (P5) cannot be tested independently of (ESR). Indeed, it would seem rather question-begging to say that (ESR) is (probably) true because it predicts the success of science. For, after all, the success of science is what demands an explanation in the first place. Second, if the success of science is a prediction made by (ESR), then (WS) seems to yield the same prediction. So, unlike in the case of the big-bang model, where the discovery of the CMB makes sense from the vantage point of the big-bang model, but not from the vantage point of the steady-state model, the success of science would make sense from the vantage point of (ESR) as well as from the vantage point of (WS). If this is correct, then (P5) does not provide a good reason to prefer (ESR) over (WS) as an explanation for the success of science.

But perhaps (ESR) does yield independently testable predictions. According to Laudan (1984, p. 109), “A theory is successful provided that it makes substantially correct predictions, that it leads to efficacious interventions in the natural order, or that it passes a suitable battery of standard tests." Accordingly, another prediction (ESR) possibly yields is the following:

(P6) If (ESR) is the best explanation for the success of science, then we would expect our interventions in nature to be effective.

The intuitively plausible idea here is that, if our interventions in nature are guided by our scientific theories, then they would fail miserably if our theories were not approximately true. For example, if our theories of biological evolution and inheritance were not even approximately true, then our attempts to fight against microbes and pests that threat to destroy our crops would fail miserably. ${ }^{15}$ The problem, however, is that (WS) seems to yield the same prediction. If (WS) and (ESR) are equally plausible explanations for the success of science, then (P6) does not give us a good reason to prefer (ESR) over (WS) as an explanation for the success of science, for (P6) is a prediction that both (ESR) and (WS) make. ${ }^{16}$

To this it might be objected that (ESR) and (WS) are not equally plausible explanations for the success of science. One might argue that (ESR) and (WS) are not equally plausible explanations for success as follows:

(O1) If $e_{1}$ logically entails $e_{2}$, then $e_{1}$ is a more plausible explanation than $e_{2}$.

(O2) (ESR) logically entails (WS).

(O3) Therefore, (ESR) is a more plausible explanation than (WS). ${ }^{17}$

Note that the relation of entailment here is supposed to hold between two explanations, not between explanandum and explanans. But why should the fact that one explanation entails another provide a good reason for thinking that the first explanation is more plausible than the

\footnotetext{
${ }^{15}$ On applied evolution, see Bull and Wichman (2001).

${ }^{16}$ Cf. Frost-Arnold (2010, p. 36) who argues that "Scientific realism (as it appears in the NMA) generates no new predictions and does not unify any apparently disparate claims."

${ }^{17}$ I am grateful to an anonymous reviewer of this journal for raising this objection.
} 
other? If to commit suicide is to kill oneself, then, strictly speaking, (AE) entails suicide. For if the man killed himself accidentally, then it follows that he killed himself. But I doubt that that would be a good reason to prefer suicide over (AE). Both suicide and (AE) seem to be equally plausible qua explanations for the man's death. And one of them could be the correct explanation independently of the other. p. 154):

Furthermore, it is not obvious to me that premise (O2) is true. According to Price (1940,

There are different degrees of as-ifness. This is acknowledged in ordinary speech, where we find such phrases as 'to some extent as if', 'rather as if', 'very much as if', 'exactly as if'.

Accordingly, it seems to me that (ESR) entails (WS) only if 'as if' means "exactly as if." However, if 'as if' means something like "to some extent as if" or "rather as if," then it is not clear that (ESR) entails (WS). For (ESR) says that mature scientific theories are approximately true, which seems to mean that the world should behave "exactly as if" those theories are true, or at least to a very high degree of 'as if', not "to some extent as if" they are true (or some other low degree of 'as if'). In other words, if there is a mismatch between the degree of as-ifness in (WS) and the degree of (approximate) truth in (ESR), then it is not clear that (ESR) entails (WS).

One might also argue that (ESR) and (WS) are not equally plausible explanations for success as follows:

(O4) If $e_{1}$ explains $e_{2}$, then $e_{1}$ is a more plausible explanation than $e_{2}$.

(O5) (ESR) explains (WS).

(O6) Therefore, (ESR) is a more plausible explanation than (WS). ${ }^{18}$

Again, note that the relation of explanation here is supposed to hold between two explanations. But why should the fact that one explanation explains another provide a good reason for thinking that the first explanation is more plausible than the other? That the battery is dead explains why my car does not start. That the engine has overheated also explains why my car does not start. That the battery is dead explains why the engine has overheated (because the cooling system needs power from the battery in order to work). But the fact that the dead battery explains the overheating of the engine does not make it a more plausible explanation for why my car does not start. Both dead battery and overheated engine seem to be plausible explanations for why my car does not start. And one of them could be the correct explanation independently of the other.

Moreover, it is not obvious to me that premise (O5) is true. Whether (ESR) explains (WS) seems to depend on the degree of as-ifness involved, as in the previous argument. Perhaps (ESR) would explain (WS) if 'as if' means "exactly as if," but it is not clear that (ESR) would explain (WS) if 'as if' means "to some extent as if." For then it would still be puzzling why the world behaves only to some extent as if (or some other low degree of 'as if') our mature theories were true if they are (approximately) true. In other words, if there is a mismatch between the

\footnotetext{
${ }^{18}$ I am grateful to an anonymous reviewer of this journal for raising this objection.
} 
degree of as-ifness in (WS) and the degree of (approximate) truth in (ESR), then it is not clear that (ESR) explains (WS).

The point is that whether one explanation entails or explains another does not necessarily bear on their plausibility qua explanations. As Campos (2009) writes:

In evaluating inductive probability, we weigh the relative support that all of the empirical data provide for one or the other hypothesis; while in evaluating explanatory plausibility, we judge how well an explanation coheres with our current system of beliefs, that is, with our background theoretical knowledge, and with what we perceive or think to be possible.

Accordingly, explanatory plausibility has to do with a hypothesis' ability to decrease the degree to which we find the explanandum surprising. In that respect, both (ESR) and (WS) seem to have some explanatory plausibility insofar as both decrease the degree to which we find the explanandum (i.e., the success of science) surprising. In other words, they both pass the first step that filters out implausible explanations. Inductive probability, on the other hand, has to do with a hypothesis' truth. What bears on a hypothesis' inductive probability, and hence on its truth, is empirical data, obtained through experimental and observational testing. In that respect, it is the independent testing of predictions that would make (ESR) more probable, rather than merely more plausible, than (WS). ${ }^{19}$

At this point, some might persist in thinking that, as an explanation for the success of science, (ESR) is clearly better than (WS). Hence, some might argue, even if these two explanations do not yield independently testable predictions that would clearly favour one over the other, we could still select (ESR) as the best explanation. It seems to me that there is not much one could say by way of a persuasive reply to this objection. Surely, scientific realists would find (ESR) intuitively better as an explanation for success, whereas anti-realists would not share this intuition. It is important to note, however, that for both realist and anti-realist explanations for success, it is a relation that is supposed to do the explanatory work. As Psillos (2001, p. 347) puts it:

Typical arguments from the success of science-be they realist or antirealist - take the weight of explanation of the success of a theory to be carried by some relation (objective: truth, empirical adequacy; or quasi-objective: the world behaves 'as if' the theory were true) between the specific theory and the world.

Accordingly, it is not obvious that an objective relation is clearly better than a quasi-objective relation as an explanation for success. Indeed, I take it as further support for my argument that we do not need to settle this question. Rather than appeal to intuitions, a better way to choose

\footnotetext{
${ }^{19}$ These remarks raise the following question: Are explanatory criteria, such as simplicity and coherence, truthconducive? This question is beyond the scope of this paper. For present purposes, my aim is to show that the NMA fails, not that IBE is an illegitimate form of inference. To that end, I set aside general problems with IBE and argue that, given that (ESR) and (WS) are equally plausible explanations for the success of science, and that they yield the same independently testable predictions, there is no good reason to prefer (ESR) over (WS), as proponents of the NMA argue.
} 
between (ESR) and (WS) is to test them. However, since they both make the same testable predictions, there is no good reason to prefer one over the other as the best explanation for the success of science. If this is correct, then we should suspend judgment as to which explanation is the best explanation for success.

\section{Conclusion}

I have argued that the NMA ultimately fails as an abductive defence of (ESR). The NMA is supposed to be an IBE that purports to explain the success of science. However, the explanation offered as the best explanation for success, namely (ESR), fails to yield independently testable predictions that alternative explanations for success do not yield. If this is correct, then there seems to be no good reason to prefer (ESR) over alternative explanations for success.

This result would probably be more worrisome to scientific realists than to anti-realists. Clearly, anti-realists do not endorse the NMA, and some of them reject IBE altogether (at least some constructive empiricists do). But for scientific realists, particularly naturalists, ${ }^{20}$ this result is worrisome because they argue that (ESR) is the best explanation for the success of science and they often point out that IBE is an actual methodology of science. ${ }^{21}$ However, given that antirealist explanations, such as (WS), are plausible explanations for the success of science, scientific realists must show that (ESR) is the best explanation. If my argument is sound, then the only way to show this is by independent testing of predictions. Then, based on whether or not those predictions are borne out, we can judge the merit of (ESR), compared with alternative explanations, as an explanation for the success of science. In that respect, the failure of the NMA might be temporary, pending further testing.

\section{Acknowledgments}

Thanks to an anonymous reviewer of this journal for helpful comments on an earlier draft of this paper. A version of this paper was presented at the Long Island Philosophical Society conference in St. Francis College, New York in April 2011. I would like to thank Glenn Statile and the audience for their helpful feedback.

\section{References}

Baggini, J. and Fosl, P. S. (2010). The Philosopher's Toolkit. (2 ${ }^{\text {nd }}$ ed.). Malden, MA: WileyBlackwell.

Bird, A. (1998). Philosophy of Science. Montreal: McGill-Queen’s University Press.

Bird, A. (2007). Inference to the Only Explanation. Philosophy and Phenomenological Research, LXXIV(2), 424-432.

Bird, A. (2010). Eliminative Abduction-Examples from Medicine. Studies in History and Philosophy of Science, 41, 345-352.

Boyd, R. (1983). The Current Status of the Issue of Scientific Realism. Erkenntnis, 19, 45-90.

\footnotetext{
${ }^{20}$ According to Psillos (1999, p. 78), "a realist epistemology of science should employ no methods other than those used by scientists themselves." Cf. Frost-Arnold (2010).

${ }^{21}$ See, e.g., Boyd (1983), Bird (1998), and Lipton (2000).
} 
Bull, J. J. and Wichman, H. A. (2001). Applied Evolution. Annual Review of Ecology and Systematics, 32, 183-217.

Campos, D. G. (2009). On the Distinction between Peirce's Abduction and Lipton's Inference to the Best Explanation. Synthese, DOI: 10.1007/s11229-009-9709-3.

Fine, A. (1986). Unnatural Attitudes: Realist and Instrumentalist Attachments to Science. Mind, 95, 149-179.

Frost-Arnold, G. (2010). The No-Miracles Argument for Realism: Inference to an Unacceptable Explanation. Philosophy of Science, 77, 35-58.

Harman, G. (1965). The Inference to the Best Explanation. Philosophical Review, 74, 88-95.

Hempel, C. G. (1958). The Theoretician’s Dilemma. In H. Feigl, M. Scriven, and G. Maxwell (Eds.), Minnesota Studies in the Philosophy of Science, Vol. II, (pp. 37-98). Minneapolis: University of Minnesota Press.

Iranzo, V. (2007). Abduction and Inference to the Best Explanation. Theoria, 60, 339-346.

Iranzo, V. (2008). Reliabilism and the Abductive Defence of Scientific Realism. Journal for General Philosophy of Science, 39, 115-120.

Kitcher, P. (1981). Explanatory Unification. Philosophy of Science, 48, 507-531.

Kitcher, P. (1983). Abusing Science: The Case Against Creationism. Cambridge, MA: The MIT Press.

Kosso, P. (1992). Reading the Book of Nature: An Introduction to the Philosophy of Science. New York: Cambridge University Press.

Kukla, A. (1998). Studies in Scientific Realism. New York: Oxford University Press.

Laudan, L. (1984). Science and Values. Berkeley: University of California Press.

Leplin, J. (1997). A Novel Defence of Scientific Realism. New York: Oxford University Press.

Lipton, P. (2000). Tracking Track Records: I. Peter Lipton. Proceedings of the Aristotelian Society, Supplementary Volume, 74, 179-205.

Lipton, P. (2004). Inference to the Best Explanation. ( $2^{\text {nd }}$ ed.). London: Routledge.

Lyons, T. D. (2003). Explaining the Success of a Scientific Theory. Philosophy of Science, 70, 891-901.

Muller, F. A. (2008). In Defence of Constructive Empiricism: Maxwell’s Master Argument and Aberrant Theories. Journal of General Philosophy of Science, 39, 131-156.

Musgrave, A. (1988). The Ultimate Argument for Scientific Realism. In R. Nola (Ed.), Relativism and Realism in Science. Dordrecht: Kluwer Academic Press.

Newman, M. (2010). The No-Miracles Argument, Reliabilism, and a Methodological Version of the Generality Problem. Synthese, 177, 111-138.

Niiniluoto, I. (2004). Truth-seeking by Abduction. In F. Stadler (Ed.), Induction and Deduction in the Sciences, (pp. 57-82). Dordrecht: Kluwer Academic Press.

Price, H. H. (1940). Hume's Theory of the External World. Oxford: Clarendon Press.

Psillos, S. (1999). Scientific Realism: How Science Tracks Truth. London: Routledge.

Psillos, S. (2001). Predictive Similarity and the Success of Science: A Reply to Stanford. Philosophy of Science, 68, 346-255.

Psillos, S. (2002). Simply the Best: A Case for Abduction. In A. C. Kakas and F. Sadri (Eds.), Computational Logic (pp. 605-625). Berlin: Springer-Verlag.

Psillos, S. (2006). Thinking about the Ultimate Argument for Realism. In C. Cheyne and J. Worrall (Eds.), Rationality \& Reality: Essays in Honour of Alan Musgrave (pp. 133-156). Dordrecht: Springer. 
Psillos, S. (2007). The Fine Structure of Inference to the Best Explanation. Philosophy and Phenomenological Research, LXXIV(2), 441-448.

Putnam, H. (1975). Mathematics, Matter and Method. Cambridge, MA: Cambridge University Press.

Sober, E. (1988). Reconstructing the Past: Parsimony, Evolution and Inference. Cambridge, MA: MIT Press.

Sober, E. (1994). Let's Razor Ockham's Razor. In E. Sober (Ed.). From a Biological Point of View (pp. 136-157). Cambridge: Cambridge University Press.

Sober, E. (1998). Simplicity (in scientific theories). In E. Craig (Ed.). Routledge Encyclopedia of Philosophy. London: Routledge. Retrieved September 27, 2010, from http://www.rep.routledge.com/article/Q095SECT1.

Sober, E. (2001). What is the Problem of Simplicity? In A. Zellner, H. Keuzenkamp, \& M. McAleer (Eds.). Simplicity, Inference, and Modeling: Keeping it Sophisticatedly Simple (pp. 13-31). Cambridge: Cambridge University Press.

Stanford, P. K. (2000). An Anti-realist Explanation of the Success of Science. Philosophy of Science, 67, 266-284.

Stanford, P. K. (2006). Exceeding Our Grasp: Science, History, and the Problem of Unconceived Alternatives. New York: Oxford University Press.

van Fraassen, B. C. (1980). The Scientific Image. New York: Oxford University Press.

Zahar, E. (1973). Why Did Einstein’s Programme Supersede Lorentz's? (I, II) British Journal for the Philosophy of Science, 24, 95-123, 223-262. 